\title{
Role of Rho kinases in abnormal and normal hematopoiesis
}

\author{
Raghuveer Singh Mali, Simryn Kapur, and Reuben Kapur \\ Herman B Wells Center for Pediatric Research, Indiana University School of Medicine, \\ Department of Pediatrics, Indianapolis, Indiana, USA
}

\begin{abstract}
Purpose of review—Rho kinases (ROCKs) are involved in regulating a variety of physiologic functions including cytoskeletal reorganization, migration, adhesion, survival and proliferation. They do so via activating several different downstream substrates such as myosin light chain phosphatase, LIM kinase and ezrin/radixin/moesin proteins. To date, most of the conclusions with regard to the function of ROCKs have involved the use of cell line models, pharmacologic inhibitors and dominant negative approaches. Importantly, the role of ROCK in hematopoiesis or leukemogenesis in the context of whole organism remains poorly understood.
\end{abstract}

Recent findings-Recent studies utilizing mice deficient in the expression of ROCK1 have begun to shed some light into the physiologic role(s) of ROCK in both normal and abnormal hematopoiesis. Findings, thus far, suggest that ROCK plays an essential role in regulating growth and survival in different hematopoietic lineages via distinct mechanisms, in part, by utilizing distinct downstream substrates including maintaining the activation of tumor-suppressor genes.

Summary-In blood cells, emerging data suggest that ROCK plays an essential role in negatively regulating inflammatory and erythropoietic stress and positively regulates the growth and survival of leukemic cells.

\section{Keywords}

erythropoiesis; hematopoietic stem cells; leukemia; Rho kinase

\section{INTRODUCTION}

Members of the Rho guanosine 5'-triphosphatase (GTPase) family play an essential role in regulating cell migration, proliferation and apoptosis [1,2]. They stimulate contractility and adhesion by generating actin stress fibers and focal adhesions in diverse cell types [3]. Rho GTPases cycle between guanosine 5'-diphosphate-bound inactive and guanosine 5'triphosphate-bound active forms and the guanosine 5'-triphosphate-bound form binds to specific targets that exert biological functions [4,5]. Two closely related Rho kinases (ROCKs), ROCK1 and ROCK2, act as downstream effectors of Rho GTPases and contribute to multiple cytoskeletal functions [6,7]. Although informative, it is important to

(C) 2014 Wolters Kluwer Health | Lippincott Williams \& Wilkins

Correspondence to Reuben Kapur, 1044 W. Walnut Street, Indianapolis, IN 46202, USA. Tel: +1 317274 4658; rkapur@iupui.edu. Conflicts of interest

There are no conflicts of interest. 
recognize that most of the conclusions with regard to ROCK have been based on studies conducted in cells of nonhematopoietic lineages. Furthermore, while ROCK1 and ROCK2 share significant sequence homology in the kinase domain (>90\%), the regulatory domains at the $\mathrm{C}$ terminus show significant divergence [8,9]. Although the functional differences between ROCK1 and ROCK2 are poorly understood, pharmacologic studies as well as studies conducted with activated and dominant active versions of ROCK in nonhematopoietic cells suggest that ROCKs contribute to increased actin-myosin IImediated contractility by directly phosphorylating myosin light chain (MLC) and negatively regulating MLC phosphatase by phosphorylating the myosin-binding subunit of MLC phosphatase [8-12]. ROCKs also activate LIM kinase, phosphorylate cofilin and inhibit its actin-depolymerization activity, leading to stabilization of actin stress fibers in fibroblasts [13-15]. How isoform-specific forms of ROCK regulate normal and abnormal blood cell development and function in the context of whole organisms is only beginning to be unraveled. Here, we briefly summarize some recent findings related to this topic and provide some future perspectives.

\section{Rho KINASES AND HUMAN EMBRYONIC STEM CELLS}

Although less is known about ROCK's role in hematopoietic stem cell (HSC) biology, recent studies show that pharmacologic inhibition of ROCK results in enhanced survival of human embryonic stem (hES) cells and enhanced survival of ROCK1 heterozygous mice in response to ultraviolet-induced stress compared with wild-type control mice [16,17]. Inhibition of ROCKs also results in reduced apoptosis of cardiomyocytes during ischemiareperfusion injury [18]. Similarly, pharmacologic inhibition of ROCK activity by ROCK inhibitor results in enhanced recovery of cryopreserved hES cells as well as induced pluripotent stem cells $[16,19]$. Furthermore, treatment with ROCK inhibitor also results in an increase in the colony number and size of hES cells [20]. In the context of hES cells, ROCK inhibitor inhibits ROCK-dependent hyperactivation of actomyosin, which is triggered by loss of E-cadherin-dependent intercellular contact [21]. Collectively, these results suggest that loss of ROCK or inhibition of ROCK activity is associated with enhanced survival of hES cells. Whether a similar pathway is also active in HSCs remains to be determined.

\section{Rho KINASE IN MYELOID CELL BIOLOGY}

Although the role of ROCK in the context of primitive HSCs remains to be investigated, recent findings have begun to shed some light into how ROCK regulates myeloid cell functions. Vemula et al. [22] investigated the role of ROCK1 in inflammatory cell migration including macrophages and neutrophils using $\mathrm{ROCKI}^{-1-}$ mice. These authors demonstrated an unexpected role for ROCK1 in negatively regulating the recruitment and migration of primary macrophages and neutrophils in vitro and in vivo in the context of whole organism. Specifically, they showed enhanced migration of both macrophages and neutrophils in response to multiple stimuli, including macrophage colony-stimulating factor (M-CSF), fibronectin, monocyte chemoattractant protein-1 and formyl-methionyl-leucylphenylalanine. The defects they observed were present in $\mathrm{ROCKI}^{-/-}$bone marrow-derived macrophages, peritoneal cavity-derived macrophages and neutrophils. Importantly, the 
observed defects were not because of the changes in the maturation and expression of $\beta 1$ integrins or because of the changes in the expression of ROCK2 in $R O C K 1^{-1-}$ cells. At the biochemical level, they showed a critical and unique role for ROCK1 in regulating the intracellular levels of phosphatidylinositol $(3,4,5)$-triphosphate/AKT in response to receptor activation by regulating the phosphorylation, degradation, stability and activity of tumorsuppressor protein phosphatase and tensin homolog (PTEN). Specifically, they showed that the serine/threonine kinase ROCK1 binds to PTEN in response to M-CSF stimulation and that this binding is enhanced when PTEN is phosphorylated. Furthermore, M-CSF-induced ROCK1 activity induces the phosphorylation of at least serine (Ser) 380, threonine (Thr) 382 and Thr383 in the PTEN carboxy tail. In the absence of ROCK1 expression, no physical association between PTEN and ROCK2 is observed. As a consequence, carboxy-terminal Ser380, Thr382 and Thr383 show significant impairment in phosphorylation, which results in enhanced degradation, reduced stability and reduced overall PTEN activity in these cells. The functional changes in $\mathrm{ROCK}^{-/-}$cells take place in spite of the presence of ROCK2, suggesting that ROCK1 functions with specificity in regulating the phosphorylation, stability and activity of PTEN and in repressing the recruitment of inflammatory cells during infection. Vemula et al.'s [22] findings are consistent with other studies showing a critical role for ROCK in regulating PTEN localization using a pharmacologic inhibitor of ROCK [23]. These studies found PTEN to colocalize with active RhoA at the posterior of the cell. Thus, it appears that at least in macrophages or inflammatory cells, ROCK1 is the principal regulator of PTEN phosphorylation on serine and threonine residues in response to M-CSF and plays an important role in regulating the stability and activity of PTEN. Importantly, deregulation of this pathway in ROCK1-deficient cells results in enhanced recruitment of macrophages in vivo. Vemula et al.'s [22] results in primary ROCK1 deficient cells demonstrating enhanced degradation and reduced stability of PTEN due to reduced phosphorylation of PTEN on serine and threonine residues are consistent with previous studies implicating these three residues in regulating PTEN stability and degradation. Using serine or threonine to alanine mutants of PTEN and overexpression systems, Vazquez et al. [24] showed that point mutations in either serine or threonine residues render less-stable PTEN. Although, the precise mechanism by which the PTEN protein in ROCK1-deficient cells is degraded is unclear; PTEN tail contains two putative PEST sequences that have been implicated in targeting proteins for proteolytic degradation. One of the putative PEST sequence includes the three residues that show defective phosphorylation of PTEN in ROCK1-deficient cells (i.e. Ser380, Thr382 and Thr383), raising the possibility that PTEN might be targeted for proteosomal or caspase-3-mediated degradation in ROCK1 cells. Given the fact that PTEN plays an essential role in regulating the self-renewal of HSCs, it will be interesting to determine whether ROCK1 also regulates PTEN activity and consequently self-renewal of HSCs.

\section{Rho KINASE IN STRESS ERYTHROPOIEISIS}

Although several downstream signaling molecules have been identified that regulate steadystate erythropoiesis, the major regulators under conditions of stress remain poorly defined. In this context, Vemula et al. [25--] have recently shown that lack ofROCK1in a phenylhydrazine-induced oxidative stress model results in enhanced recovery from 
hemolytic anemia as well as enhanced stress erythropoiesis compared with control mice. Deficiency of ROCK1 also results in enhanced survival, whereas wild-type mice die rapidly in response to stress. Enhanced survivability of ROCK1-deficient mice is associated with reduced level of reactive oxygen species. Bone marrow transplantation studies revealed that enhanced stress erythropoiesis in ROCK1-deficient mice is stem cell autonomous.

Biochemically, ROCK1 uniquely regulates the expression of p53 in response to oxidative stress by regulating its phosphorylation. Interestingly, ROCK1 physically associates with p53 in normal splenocytes in response to oxidative stress. In the absence of this interaction, reduced caspase- 3 cleavage and reduced level of reactive oxygen species are observed. Prior studies have shown that loss of p53 in p53-deficient mice also results in enhanced erythroid cell recovery following an oxidative challenge [26].Thus, these studies reveal a novel mechanism of 553 regulation in stress erythropoiesis. Given the fact that p53 levels are often upregulated in erythroid progenitors derived from patients with Diamond-Blackfan anemia, which is associated with enhanced apoptosis and reduced cell cycle, it will be interesting to determine whether inhibition of ROCK1 expression in these cells will rescue the survival and associated cell cycle defects in cells harboring mutations in the ribosomal protein subunit 19 [27]. Taken together, studies described in ROCK1-deficient mice, thus far, suggest that ROCK1 may play an essential role in negatively regulating the survival of multiple hematopoietic lineages in the context of both inflammatory as well as oxidative stress in myeloid and erythroid cells, respectively, in part by regulating the activation of tumor-suppressor genes such as PTEN and p53. It remains to be seen whether this is true for other lineages as well.

\section{Rho KINASE IN LEUKEMOGENESIS}

Although the role of ROCK in solid tumor metastasis has been well established, its role in the context of leukemogenesis is only beginning to be unraveled [28]. Small molecule inhibitors of ROCK, namely fasudil (HA-1077), Y27632 and H-1152P or dimethylfasudil, have been used to assess the role of ROCK in leukemogenesis [28]. These inhibitors inhibit the activity of both ROCK1 and ROCK2 in an ATP-competitive manner. Of the four most well described ROCK inhibitors, fasudil is the only one currently used in humans for the treatment of cardiovascular diseases such as hypertension, angina and stroke. Mali et al. [28] recently demonstrated constitutive activation of ROCK in leukemic cells expressing activating forms of KIT, FMS-like tyrosine kinase 3 and breakpoint cluster region-abelson kinase, which are oncogenes commonly found in patients with systemic mastocytosis, acute myeloid leukemia (AML) and chronic myeloid leukemia, respectively. All three oncogenes are associated with poor prognosis and survival in these patients. Mali et al. [28] showed that either loss of ROCK1 in HSCs bearing the oncogenes described above or pharmacologic inhibition of ROCK in leukemic cells inhibited the growth and survival of leukemic cells in vitro and, importantly, prolonged the lifespan of leukemic mice in vivo. Although Vemula et al. [25--] found that in the context of stress responses ROCK1 regulates PTEN and p53 activation and negatively impacts stress hematopoiesis, in the context of leukemogenesis Mali et al. [28] found an essential role for MLC. They found MLC to be constitutively hyperphosphorylated on Ser19 in leukemic cells, whose activation could be rapidly inhibited upon treating the leukemic cells with ROCK inhibitors. Importantly, 
increased MLC phosphorylation in leukemic cells was associated with enhanced actin polymerization, which was inhibited in the presence of ROCK inhibitors and associated with rapid F-actin depolymerization followed by membrane blebbing and rapid cell death. This phenomenon was somewhat unique to leukemic cells; thus, it is likely that MLC may be uniquely involved in the growth of leukemic cells but not normal hematopoietic cells as further shown by MLC knockdown experiments [28].

Although ROCK inhibitors are good initial tools to dissect the role of ROCK in leukemic blasts, further experiments utilizing mice deficient in the expression of ROCK1, ROCK2 and both ROCK1 and ROCK2 will be needed to more precisely delineate the role of ROCK in initiation and progression of leukemia. In addition, it will be useful to assess whether the traditional well-characterized substrates of ROCK, such as LIM kinase and Ezrin, also contribute to leukemogenesis via the oncogenes described above. Small molecule inhibitors and mice deficient in the expression of these kinases have been described and will likely function as useful tools to dissect their respective role(s) in the context of leukemogenesis. An additional important question that remains to be answered relates to the involvement of ROCK1 and ROCK2 in regulating the growth and survival as well as actin-based functions in leukaemia-initiating cells or leukemia stem cells as well as their role in regulating drugresistant mutations of breakpoint cluster region-abelson kinase in chronic myeloid leukemia and FMS-like tyrosine kinase 3-internal tandem duplication in AML. Some studies in this area are already being conducted. Recent studies involving an unbiased genome-wide pooled short hairpin ribonucleic acid screen in primary human AML cells revealed that knockdown of ROCK1 in human primary leukemic blasts results in rapid cell cycle arrest and cell death. Treatment with fasudil proved to be equally effective in killing leukemic cells in these studies. Importantly, fasudil was less toxic to normal hematopoietic cells compared to primary leukemic cells. Fasudil treatment of these cells suggested that it had a significant negative effect on the self-renewal capacity of primary human leukemic stem/ progenitor cells [29-].

Once a better understanding of the individual role(s) of ROCK isoforms in normal hematopoiesis and in leukemogenesis is established, the development of isoform-specific ROCK inhibitors may be a worthwhile strategy, in light of the fact that some patients with benign hematologic issues such as Diamond-Blackfan anemia may respond better to ROCK1 inhibitors versus patients with malignant hematologic diseases that may respond better to ROCK1 and/or ROCK2 inhibitors.

\section{CONCLUSION}

Although several substrates of ROCK have been identified in cells of nonhematopoietic lineages, few have been identified in primary hematopoietic cells. ROCK isoforms are likely to regulate functions in different cell types via distinct and in some cases perhaps overlapping substrates. It is, therefore, important to assess the role of ROCK isoforms in the context of a specific cell type and in primary cells. Likewise, it would be interesting to determine whether the classical ROCK substrates, such as LIM kinase and/or Ezrin's loss of function in specific hematopoietic lineages, will result in similar functional defects as ROCK1 and/or ROCK2-deficient cells. An additional key question is to determine which 
ROCK substrates are critical for normal hematopoietic function and which are relevant for leukemogenesis. More importantly, can this information be used to differentially target molecules in leukemogenesis versus normal hematopoietic functions? A better understanding of the biology of these kinases and their substrates is likely to result in better strategies for targeting these related yet distinct molecules in different hematologic diseases.

\section{REFERENCES AND RECOMMENDED READING}

Papers of particular interest, published within the annual period of review, have been highlighted as:

- of special interest

- of outstanding interest

1. Ridley A. Rac and Rho. Curr Biol. 1999; 9:R156. [PubMed: 10074463]

2. Ridley AJ. Rho proteins, PI 3-kinases, and monocyte/macrophage motility. FEBS Lett. 2001; 498:168-171. [PubMed: 11412850]

3. Ridley A. Rho GTPases. Integrating integrin signaling. J Cell Biol. 2000; 150:F107-F109. [PubMed: 10953018]

4. Ridley AJ. The GTP-binding protein Rho. Int J Biochem Cell Biol. 1997; 29:1225-1229. [PubMed: 9451818]

5. Etienne-Manneville S, Hall A. Rho GTPases in cell biology. Nature. 2002; 420:629-635. [PubMed: 12478284]

6. Ishizaki T, Naito M, Fujisawa K, et al. p160ROCK, a Rho-associated coiled-coil forming protein kinase, works downstream of Rho and induces focal adhesions. FEBS Lett. 1997; 404:118-124. [PubMed: 9119047]

7. Riento K, Ridley AJ. Rocks: multifunctional kinases in cell behaviour. Nat Rev Mol Cell Biol. 2003; 4:446-456. [PubMed: 12778124]

8. Matsui T, Amano M, Yamamoto T, et al. Rho-associated kinase, a novel serine/threonine kinase, as a putative target for small GTP binding protein Rho. EMBO J. 1996; 15:2208-2216. [PubMed: 8641286]

9. Nakagawa O, Fujisawa K, Ishizaki T, et al. ROCK-I and ROCK-II, two isoforms of Rho-associated coiled-coil forming protein serine/threonine kinase in mice. FEBS Lett. 1996; 392:189-193. [PubMed: 8772201]

10. Amano M, Ito M, Kimura K, et al. Phosphorylation and activation of myosin by Rho-associated kinase (Rho-kinase). J Biol Chem. 1996; 271:20246-20249. [PubMed: 8702756]

11. Kawano Y, Fukata Y, Oshiro N, et al. Phosphorylation of myosin-binding subunit (MBS) of myosin phosphatase by Rho-kinase in vivo. J Cell Biol. 1999; 147:1023-1038. [PubMed: 10579722]

12. Kureishi Y, Kobayashi S, Amano M, et al. Rho-associated kinase directly induces smooth muscle contraction through myosin light chain phosphorylation. J Biol Chem. 1997; 272:12257-12260. [PubMed: 9139666]

13. Amano M, Chihara K, Kimura K, et al. Formation of actin stress fibers and focal adhesions enhanced by Rho-kinase. Science. 1997; 275:1308-1311. [PubMed: 9036856]

14. Arber S, Barbayannis FA, Hanser H, et al. Regulation of actin dynamics through phosphorylation of cofilin by LIM-kinase. Nature. 1998; 393:805-809. [PubMed: 9655397]

15. Maekawa M, Ishizaki T, Boku S, et al. Signaling from Rho to the actin cytoskeleton through protein kinases ROCK and LIM-kinase. Science. 1999; 285:895-898. [PubMed: 10436159]

16. Watanabe K, Ueno M, Kamiya D, et al. A ROCK inhibitor permits survival of dissociated human embryonic stem cells. Nat Biotechnol. 2007; 25:681-686. [PubMed: 17529971]

17. Ongusaha PP, Qi HH, Raj L, et al. Identification of ROCK1 as an upstream activator of the JIP-3 to JNK signaling axis in response to UVB damage. Sci Signal. 2008; 1:ra14. [PubMed: 19036714] 
18. Bao W, Hu E, Tao L, et al. Inhibition of Rho-kinase protects the heart against ischemia/reperfusion injury. Cardiovasc Res. 2004; 61:548-558. [PubMed: 14962485]

19. Hong H, Takahashi K, Ichisaka T, et al. Suppression of induced pluripotent stem cell generation by the p53-p21 pathway. Nature. 2009; 460:1132-1135. [PubMed: 19668191]

20. Claassen DA, Desler MM, Rizzino A. ROCK inhibition enhances the recovery and growth of cryopreserved human embryonic stem cells and human induced pluripotent stem cells. Molecular reproduction and development. 2009; 76:722-732. [PubMed: 19235204]

21. Ohgushi M, Matsumura M, Eiraku M, et al. Molecular pathway and cell state responsible for dissociation-induced apoptosis in human pluripotent stem cells. Cell Stem Cell. 2010; 7:225-239. [PubMed: 20682448]

22. Vemula S, Shi J, Hanneman P, et al. ROCK1 functions as a suppressor of inflammatory cell migration by regulating PTEN phosphorylation and stability. Blood. 2010; 115:1785-1796. [PubMed: 20008297]

23. Li Z, Dong X, Wang Z, et al. Regulation of PTEN by Rho small GTPases. Nat Cell Biol. 2005; 7:399-404. [PubMed: 15793569]

24. Vazquez F, Ramaswamy S, Nakamura N, Sellers WR. Phosphorylation of the PTEN tail regulates protein stability and function. Mol Cell Biol. 2000; 20:5010-5018. [PubMed: 10866658]

25. Vemula S, Shi J, Mali RS, et al. ROCK1 functions as a critical regulator of stress erythropoiesis and survival by regulating p53. Blood. 2012; 120:2868-2878. [PubMed: 22889758] This study describes a novel role for ROCK1 in regulating stress erythropoiesis by regulating the activation and stability of tumor suppressor p53.

26. Ganguli G, Back J, Sengupta S, Wasylyk B. The p53 tumour suppressor inhibits glucocorticoidinduced proliferation of erythroid progenitors. EMBO Rep. 2002; 3:569-574. [PubMed: 12034755]

27. Ebert BL, Lee MM, Pretz JL, et al. An RNA interference model of RPS19 deficiency in DiamondBlackfan anemia recapitulates defective hematopoiesis and rescue by dexamethasone: identification of dexamethasone-responsive genes by microarray. Blood. 2005; 105:4620-4626. [PubMed: 15755903]

28. Mali RS, Ramdas B, Ma P, et al. Rho kinase regulates the survival and transformation of cells bearing oncogenic forms of KIT, FLT3, and BCR-ABL. Cancer Cell. 2011; 20:357-369. [PubMed: 21907926]

29. Wermke M, Camgoz A, Paszkowski-Rogacz M, et al. A genome wide shRNA screen in primary human AML cells identifies ROCK1 as a novel therapeutic target. Blood. 2013; 122:170. [PubMed: 23673857] This study describes the role of ROCK1 inhibition in leukemic stem cells. 


\section{KEY POINTS}

- ROCK1 negatively regulates stress hematopoiesis, in part, by regulating the activation of tumor-suppressor genes such as PTEN and p53.

- ROCK is constitutively activated in leukemic cells and its inhibition results in cell death of leukemic cells partly via regulating the activation of MLC.

- ROCK regulates cellular functions in various hematopoietic lineages via distinct mechanisms. 